\title{
Quantum error correction beyond qubits
}

\author{
Takao Aoki ${ }^{1 \star}$, Go Takahashi ${ }^{1,2}$, Tadashi Kajiya ${ }^{1,2}$, Jun-ichi Yoshikawa ${ }^{1,2}$, Samuel L. Braunstein ${ }^{3}$, \\ Peter van Loock ${ }^{4}$ and Akira Furusawa ${ }^{1,2 \uparrow}$
}

Quantum computation and communication rely on the ability to manipulate quantum states robustly and with high fidelity. To protect fragile quantum-superposition states from corruption through so-called decoherence noise, some form of error correction is needed. Therefore, the discovery of quantum error correction $^{1,2}$ (QEC) was a key step to turn the field of quantum information from an academic curiosity into a developing technology. Here, we present an experimental implementation of a QEC code for quantum information encoded in continuous variables, based on entanglement among nine optical beams ${ }^{3}$. This nine-wave-packet adaptation of Shor's original nine-qubit scheme $^{1}$ enables, at least in principle, full quantum error correction against an arbitrary single-beam error.

Quantum error correction protocols eliminate uncontrolled errors that affect fragile quantum-superposition states by encoding these quantum states into a larger, multi-partite entangled system. Errors occurring on a limited number of parties will leave the entanglement intact and so the original state may be retrieved by error syndrome recognition followed by recovery operations. Shor ${ }^{1}$ proposed a concatenated quantum code to protect against arbitrary single-qubit errors, by encoding an arbitrary single-qubit state $|\psi\rangle=\alpha|0\rangle+\beta|1\rangle$ into nine physical qubits

$$
\left|\psi_{\text {encode }}\right\rangle=\alpha|+,+,+\rangle+\beta|-,-,-\rangle
$$

with $| \pm\rangle=(|0,0,0\rangle \pm|1,1,1\rangle) / \sqrt{2}$. Although reminiscent of the redundant encoding in classical error correction, the quantum code exhibits some clearly non-classical features of which the most significant is the presence of multi-party entanglement. The concatenation of three-party entangled states $(| \pm\rangle)$ into nine-party states enables one to correct both bit-flip and phase-flip errors. The latter type of error occurs only in non-classical states. Remarkably, suitable error syndrome measurements would collapse an arbitrary error (including coherent superpositions of bit-flip and phase-flip errors) into the discrete set of only bit-flip and/or phase-flip errors. These discrete (Pauli) errors can be easily reversed to recover the original state.

The continuous-variable version of Shor's nine-qubit code $e^{1,3}$ is the only code so far that can be deterministically (unconditionally) implemented using only linear operations and resources. Like the discrete Shor code, it can correct arbitrary errors on single channels; however, more sophisticated codes would be required to correct some important forms of error such as loss on all channels simultaneously ${ }^{4,5}$. Our experiment is the first implementation of a Shor-type code, as the preparation of nineparty entanglement is still beyond the scope of existing non-optical

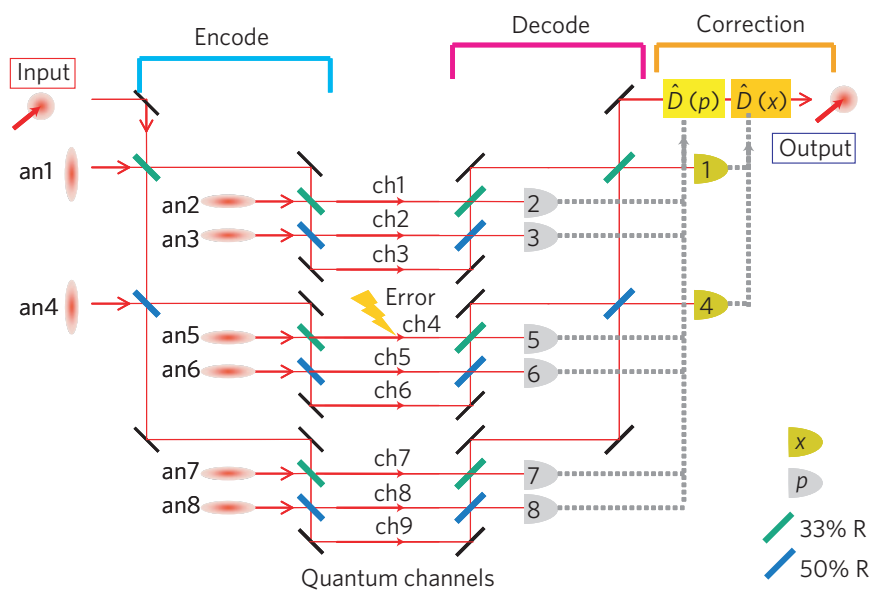

Figure 1 | Schematic diagram for the nine-wave-packet quantum error-correction code operation (ref. 3) for correcting an arbitrary error occurring in any one of the nine channels. The grey dotted lines represent the classical information that is used to compute the necessary syndrome recovery operations. an1-an8: ancillae; $R$ : reflectivity.

approaches and single-photon-based optical schemes. Indeed, previous implementations of QEC were based on qubit codes, either in liquid-state NMR (using up to five qubits) ${ }^{6-9}$, linear ion trap hardware configurations (using up to three qubits) ${ }^{10}$ or single-photon linear optics (using up to four qubits) ${ }^{11,12}$. Here, continuous-variable QEC (refs 13, 14) uses squeezed states of light and networks of beam splitters ${ }^{3}$. Even this optical approach requires an optical network three times the size of that used in earlier experiments ${ }^{15}$ to achieve the large-scale multi-partite entanglement for a nine-wave-packet code.

In our scheme, as for the simplest QEC codes (whether for qubits or for continuous variables), a single, arbitrary error can be corrected. Such schemes typically assume that errors occur stochastically and therefore rely on the low frequency of multiple errors. Stochastic error models may describe, for example, stochastic, depolarizing channels for qubits, or in the continuous-variable regime ${ }^{16}$, free-space channels with atmospheric fluctuations causing beam jitter, as considered recently for various non-deterministic distillation protocols ${ }^{17-20}$. For the continuous-variable QEC protocols, as realized in the present work, this type of error may be suppressed in a deterministic fashion (see Supplementary Information, Part F). The overall performance of this family of QEC codes is then limited only by the accuracy with which ancilla-state preparation, encoding and decoding circuits,

\footnotetext{
${ }^{1}$ Department of Applied Physics and Quantum Phase Electronics Center, School of Engineering, The University of Tokyo, 7-3-1 Hongo, Bunkyo-ku, Tokyo 113-8656, Japan, ${ }^{2}$ CREST, Japan Science and Technology (JST) Agency, 5, Sanbancho, Chiyoda-ku, Tokyo 102-0075, Japan, ${ }^{3}$ Computer Science, University of York, York YO10 5DD, UK, ${ }^{4}$ Optical Quantum Information Theory Group, Max Planck Institute for the Science of Light and Institute of Theoretical Physics I, Universität Erlangen-Nürnberg, Staudtstr.7/B2, 91058 Erlangen, Germany. ${ }^{\star}$ Current address: Department of Physics, Graduate School of Science, Kyoto University, Kyoto 606-8502, Japan. †e-mail: akiraf@ap.t.u-tokyo.ac.jp.
} 
Table 1 | Error syndrome measurements.

\begin{tabular}{lll}
$\begin{array}{l}\text { Channel with } \\
\text { an error }\end{array}$ & $\begin{array}{l}\text { Detectors with } \\
\text { non-zero outputs }\end{array}$ & $\begin{array}{l}\text { LO } \\
\text { phase }\end{array}$ \\
\hline 1 & 1 & $x$ \\
& 2 & $p$ \\
2 & 1 & $x$ \\
& $2,3(\mathrm{DS})$ & $p$ \\
3 & 1 & $x$ \\
& $2,3(\mathrm{ES})$ & $p$ \\
4 & $1,4(\mathrm{DS})$ & $x$ \\
& 5 & $p$ \\
5 & $1,4(\mathrm{DS})$ & $x$ \\
& $5,6(\mathrm{DS})$ & $p$ \\
6 & $1,4(\mathrm{DS})$ & $x$ \\
& $5,6(\mathrm{ES})$ & $p$ \\
7 & $1,4(\mathrm{ES})$ & $x$ \\
& 7 & $p$ \\
8 & $1,4(\mathrm{ES})$ & $x$ \\
& $7,8(\mathrm{DS})$ & $p$ \\
9 & $1,4(\mathrm{ES})$ & $x$ \\
& $7,8(\mathrm{ES})$ & $p$ \\
\hline
\end{tabular}

LO phase: quadrature at which the local oscillator phase of the homodyne detector is locked, ES equal signs, DS: different signs.

and syndrome extraction and recovery operations can be achieved. In the continuous-variable scheme, all of these ingredients can be highly efficiently implemented. In the absence of squeezing, the fidelity is limited by the vacuum noise. We dub this case quantumlimited error correction. Squeezing of the auxiliary modes is linked with the presence of entanglement and thus determines whether the transfer fidelities exceed those of the quantum-limited error correction (see Supplementary Information, Parts E and F).

We begin with a description of the scheme in the limit of infinite squeezing, where the position $x$ and momentum $p$ of a harmonic oscillator (corresponding to a single optical mode of the light field) serve as the conjugate pair of observables used for the encoding

$$
\left|\psi_{\text {encode }}\right\rangle=\int \mathrm{d} p \psi(p)|p, p, p\rangle
$$

with $|p\rangle=(1 / \sqrt{\pi}) \int \mathrm{d} x \mathrm{e}^{2 i x p}|x, x, x\rangle$, for the units-free choice $\hbar=1 / 2$. Through this nine-wave-packet code, an arbitrary singlemode state $|\psi\rangle=\int \mathrm{d} x \psi(x)|x\rangle$ is encoded into nine optical modes. This perfectly encoded state is obtained by using eight infinitely squeezed ancilla states. Finite squeezing of the ancillae leads to an approximate encoding, and hence lowers the fidelity of the QEC.

Figure 1 shows a schematic diagram of our realization of the nine-wave-packet code. In the encoding stage, an input state is entangled with eight squeezed ancillae, each corresponding to an approximate ' 0 ' ('blank') state. After an error is introduced, the states are decoded simply by inverting the encoding. The eight ancilla modes are then measured (with $x$-quadrature measurements carried out in detectors 1 and 4 and $p$-quadrature measurements in six other detectors), and the results of the measurements are used for error syndrome recognition. More precisely, these are the results of homodyne detection applied to the ancilla modes along their initial squeezing direction.

The encoding stage consists of two steps to realize the concatenation of position and momentum codes ${ }^{3}$. First, positionencoding is achieved by means of a tritter $T_{\text {in,an1,an4 }}$, that is, two beam splitters (blue and green in Fig. 1) acting on the input mode and two $x$-squeezed ancilla modes (an1 and an 4 in Fig. 1). The second step provides the momentum-encoding by means of three more tritters, with six extra $p$-squeezed ancilla modes (an2, an3, an5, an6, an7 and an8 in Fig. 1). The overall encoding circuit becomes

$$
T_{\text {an4,an7,an8 }} T_{\text {an1,an5,an6 }} T_{\text {in,an2,an3 }} T_{\text {in,an1,an4 }}
$$

In the experiment, we generated a code state with position $x$ and momentum $p$ interchanged. This alternate encoding (and the corresponding QEC protocol) involves only a change of basis with no drop in performance. Quantum optically, this change corresponds to a $90^{\circ}$ rotation of the quadrature amplitudes, requiring local oscillator phases to be shifted by $90^{\circ}$ for homodyne detection.

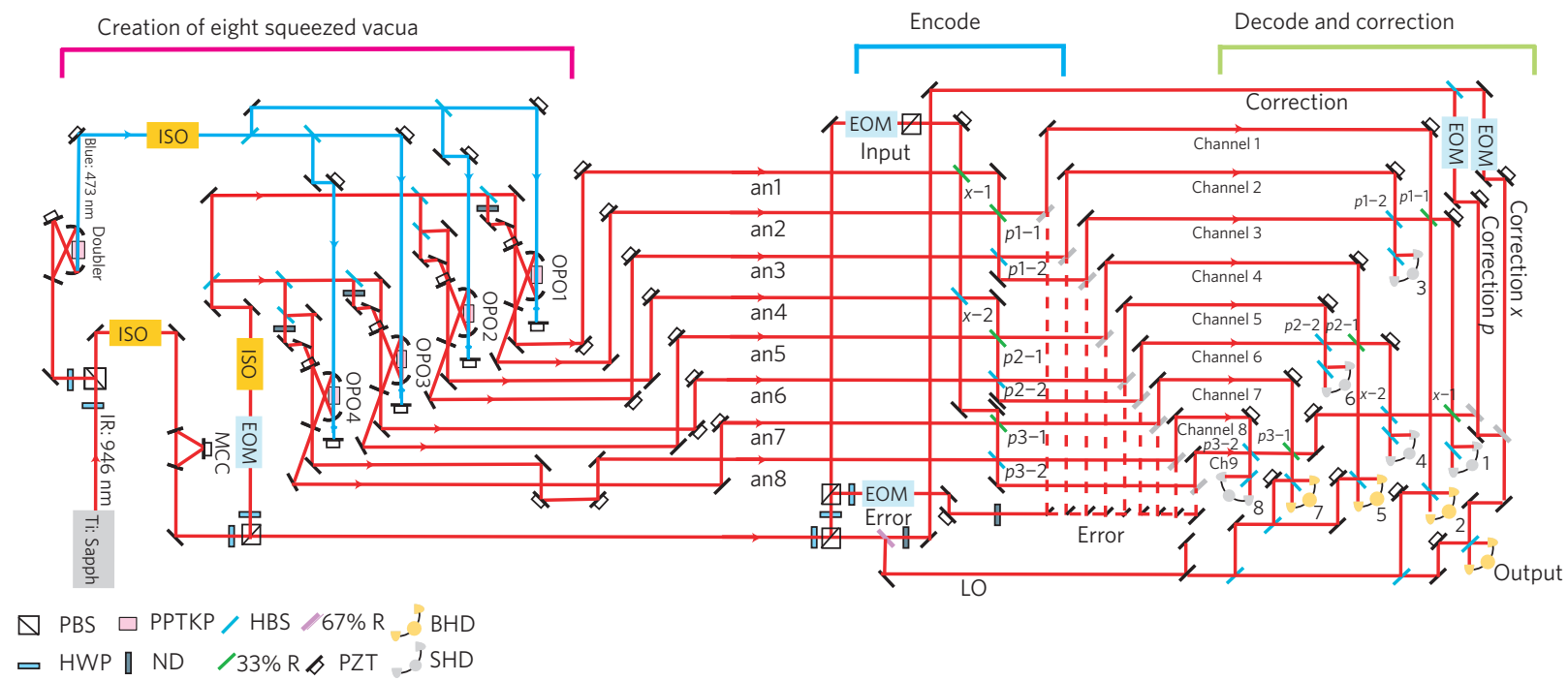

Figure 2 | Experimental set-up of the nine-wave-packet quantum error correction. PBS: polarization beam splitter, PPKTP: periodically poled KTiOPO 4 , HBS: half (symmetric) beam splitter, HWP: half-wave plate, ND: neutral density filter, PZT: piezoelectric transducer, BHD: balanced homodyning, SHD: self-homodyning, OPO: optical parametric oscillator, MCC: mode-cleaning cavity, LO: local oscillator, ISO: optical isolator, EOM: electro-optic modulator, $R$ : reflectivity. 
a

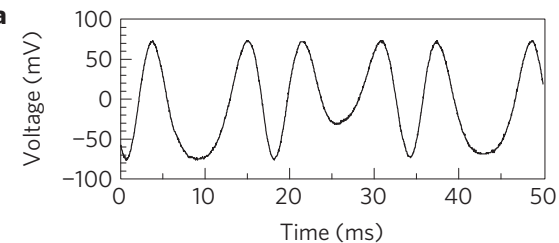

b

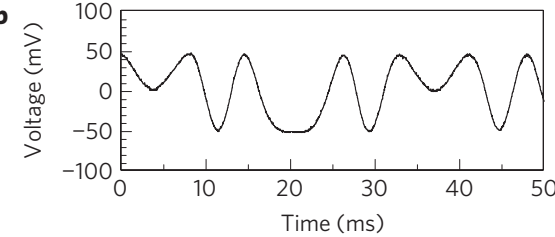

c
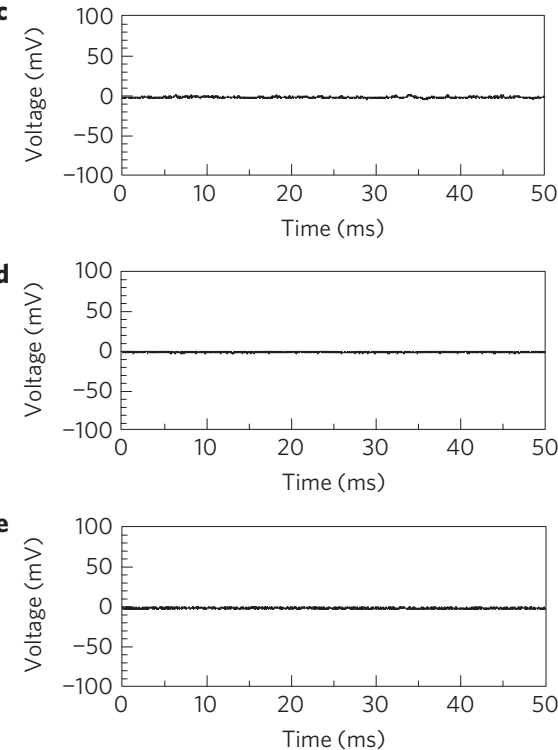

f

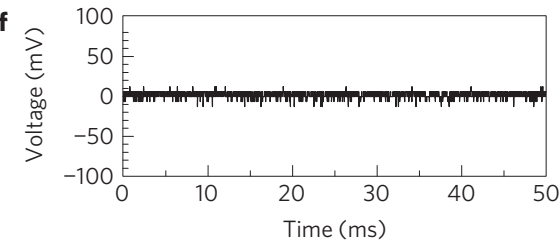

g
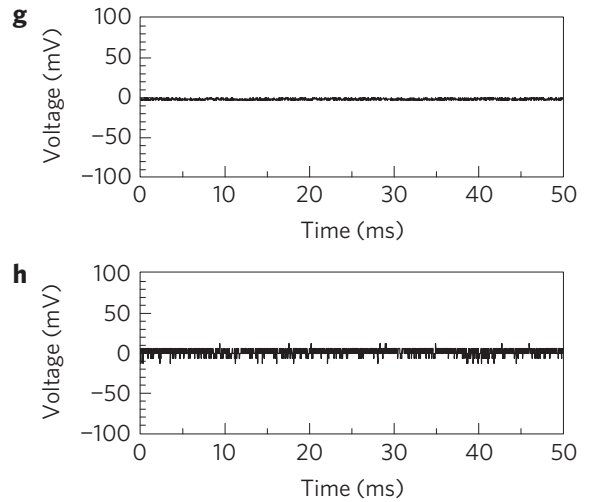
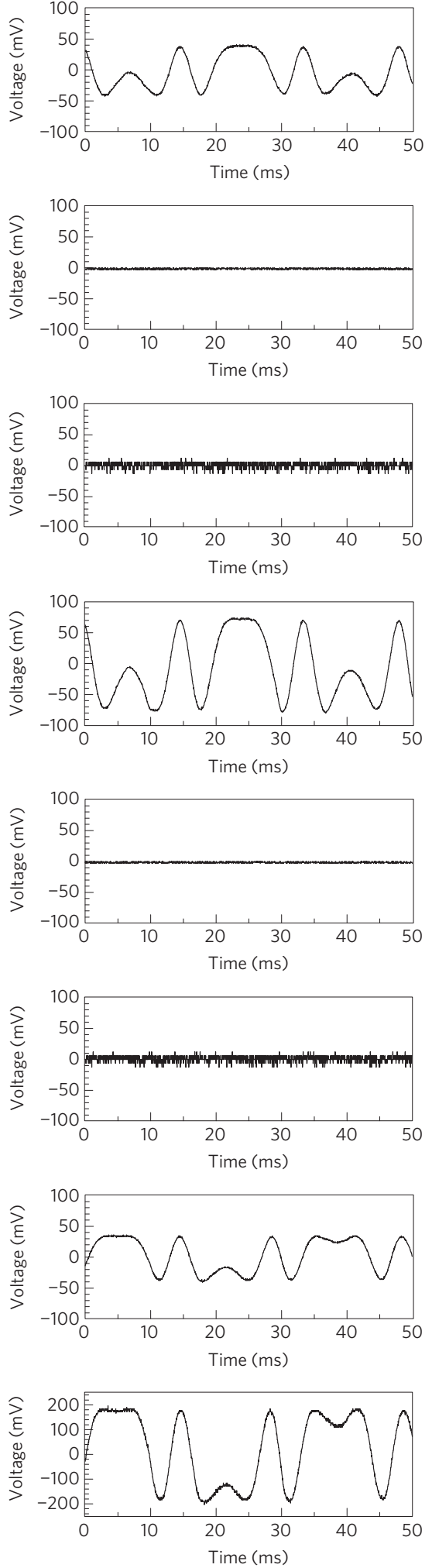

Figure 3 | Error syndrome measurement results. Left column: a random displacement error is imposed on channel 1. Right column: a random displacement error is imposed on channel 9. A two-channel oscilloscope is used to measure the outputs of detectors 1 and 4,2 and 3,5 and 6 and 7 and 8 . $\mathbf{a}-\mathbf{h}$, Output signal of detectors 1-8.

As the decoding stage merely inverts the encoding, the eight ancilla modes will remain all ' 0 ' in the absence of errors. In the presence of an error in any one of the nine channels, the measurement results of the decoded ancillae will lead to non-zero components, containing sufficient information for identifying and hence correcting the error (see Supplementary Information for derivations and Table 1 for an error-syndrome map). Similar to the qubit QEC scheme, where the conditional state after the syndrome measurements becomes the original input state up to some discrete Pauli errors, our conditional state coincides with the input state 
a

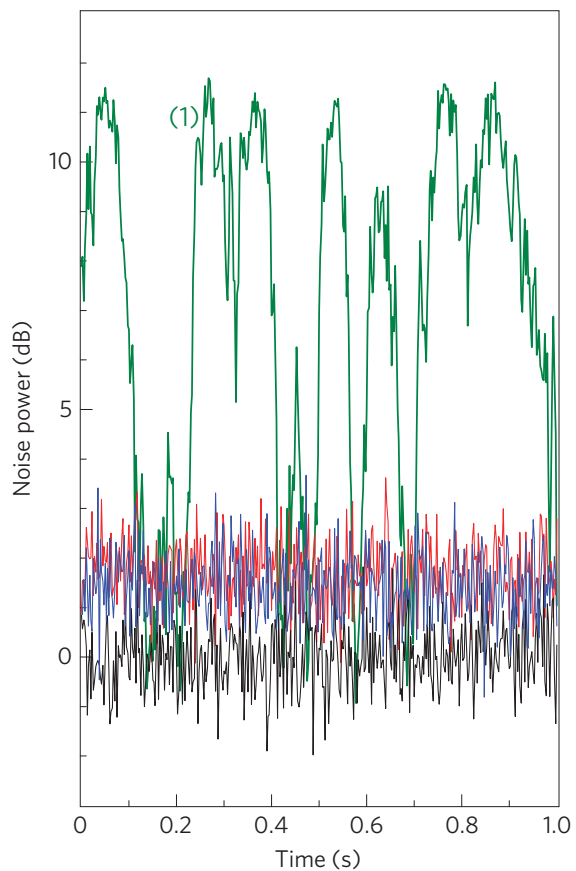

b

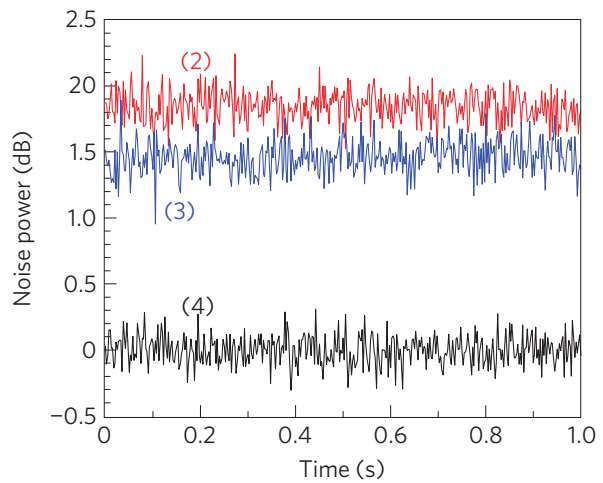

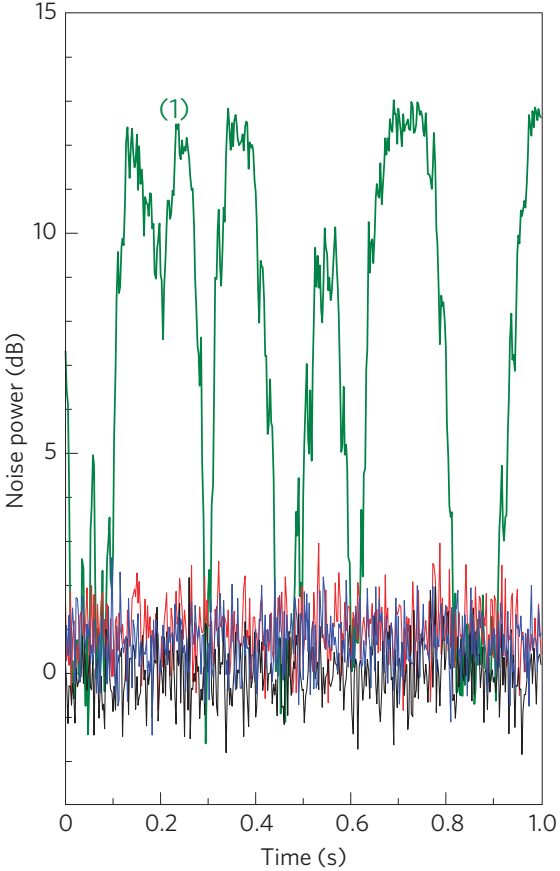

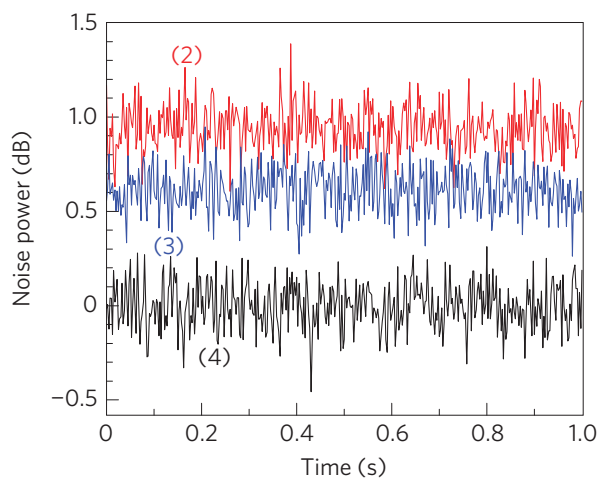

Figure 4 | Results of quantum-error-correction procedure. a, Single scan of a spectrum analyser with zero span mode (2 MHz centre frequency, $30 \mathrm{kHz}$ resolution bandwidth and $300 \mathrm{~Hz}$ video bandwidth), when a random phase-space displacement error is imposed on channel 1 (left) or channel 9 (right). For the former (latter), the local oscillator phase of the homodyne detector is locked to the $x(p)$ quadrature. In each case, four traces are shown for comparison: (1) homodyne detector output without error correction (no feed-forward step); (2) error correction output without squeezing; (3) error correction output with squeezing; (4) shot-noise level. b, Traces 2-4 averaged over 30 experiments.

up to some simple phase-space displacements. Thus, it remains only to apply the appropriate (inverse) displacement operations to correct the errors.

The detailed experimental set-up for our nine-wave-packet QEC scheme is shown in Fig. 2. Eight squeezed vacua are created by four optical parametric oscillators (OPOs), which have two counter-propagating modes; thus, every OPO creates two individual squeezed vacua. The squeezing level of each singlemode squeezed vacuum state corresponds to roughly $1 \mathrm{~dB}$ below shot noise. For pumping the OPOs, the second harmonic of a continuous-wave Ti:sapphire laser output is used. The syndrome measurements are carried out by means of homodyne detection with near-unit efficiency.

To apply a single error, a coherent modulation is first generated in a so-called error beam using an electro-optic modulator (EOM) ('modulated mode'). This beam is then superimposed onto the selected mode or channel ('target mode') through a high-reflectivity beam splitter ${ }^{21}$ with independently swept phase, resulting in a quasi-random displacement error. The errorcorrecting displacement operations (as determined by decoding and measurement) are then carried out similarly, by means of an EOM and a high-reflectivity beam splitter, but now with phase locking between the modulated and target modes along either the $x$ or $p$ axis, as appropriate.

Figure 3 shows some examples for error syndrome measurement results. Here, the input state is chosen to be a vacuum state. A random displacement error in phase space is imposed on channel 1 (Fig. 3, left column) and on channel 9 (Fig. 3, right column). A two-channel oscilloscope is used to measure the outputs of pairs of detectors $(1,4),(2,3),(5,6)$ and $(7,8)$. Comparing the results of Fig. 3, left column, with Table 1, one can identify an error occurring in channel 1 , because only detectors 1 and 2 have non-zero outputs. The outputs from detectors 1 and 2 correspond to the desired $x$ and $p$ displacements, respectively. Similarly, from Fig. 3, right column, we can recognize that an error has occurred in channel 9. Here, detectors 1, 4, 7 and 8 have non-zero outputs and the outputs of detectors 1 and 4, as well as 7 and 8 have equal signs (distinguishing it from the case of an error in channel 8, for which outcomes 7 and 8 have different signs).

Figure 4 shows two examples of QEC results, comparing output states with and without error correction, and with and without squeezing of the ancilla modes. In Fig. 4, left column, an error 


\begin{tabular}{|c|c|c|c|c|}
\hline $\begin{array}{l}\text { Error } \\
\text { on } \\
\text { mode } \\
\end{array}$ & $\begin{array}{l}\text { Quadrature } \\
\text { of output }\end{array}$ & $\begin{array}{l}\text { Output power } \\
\text { without SQ } \\
\text { (theory) }\end{array}$ & $\begin{array}{l}\text { Output power } \\
\text { without SQ } \\
\text { (experiment) }\end{array}$ & $\begin{array}{l}\text { Output power } \\
\text { with SQ } \\
\text { (experiment) }\end{array}$ \\
\hline \multirow[t]{2}{*}{1} & $x$ & 1.76 & $1.84 \pm 0.12$ & $1.46 \pm 0.13$ \\
\hline & $p$ & 0.67 & $0.68 \pm 0.12$ & $0.57 \pm 0.12$ \\
\hline \multirow[t]{2}{*}{2} & $x$ & 1.76 & $1.75 \pm 0.12$ & $1.42 \pm 0.13$ \\
\hline & $p$ & 0.87 & $0.97 \pm 0.12$ & $0.72 \pm 0.12$ \\
\hline \multirow[t]{2}{*}{3} & $x$ & 1.76 & $1.83 \pm 0.12$ & $1.41 \pm 0.12$ \\
\hline & $p$ & 0.87 & $0.92 \pm 0.12$ & $0.70 \pm 0.12$ \\
\hline \multirow[t]{2}{*}{4} & $x$ & 2.22 & $2.26 \pm 0.12$ & $1.67 \pm 0.12$ \\
\hline & $p$ & 0.67 & $0.73 \pm 0.12$ & $0.50 \pm 0.12$ \\
\hline \multirow[t]{2}{*}{5} & $x$ & 2.22 & $2.33 \pm 0.12$ & $1.79 \pm 0.12$ \\
\hline & $p$ & 0.87 & $0.88 \pm 0.12$ & $0.73 \pm 0.13$ \\
\hline \multirow[t]{2}{*}{6} & $x$ & 2.22 & $2.34 \pm 0.12$ & $1.77 \pm 0.12$ \\
\hline & $p$ & 0.87 & $0.87 \pm 0.13$ & $0.73 \pm 0.13$ \\
\hline \multirow[t]{2}{*}{7} & $x$ & 2.22 & $2.30 \pm 0.13$ & $1.72 \pm 0.12$ \\
\hline & $p$ & 0.67 & $0.69 \pm 0.12$ & $0.57 \pm 0.12$ \\
\hline \multirow[t]{2}{*}{8} & $x$ & 2.22 & $2.18 \pm 0.13$ & $1.79 \pm 0.13$ \\
\hline & $p$ & 0.87 & $0.84 \pm 0.12$ & $0.65 \pm 0.12$ \\
\hline \multirow[t]{2}{*}{9} & $x$ & 2.22 & $2.18 \pm 0.14$ & $1.82 \pm 0.13$ \\
\hline & $p$ & 0.87 & $0.94 \pm 0.12$ & $0.61 \pm 0.12$ \\
\hline
\end{tabular}

Perfect error correction therefore corresponds to $\mathrm{O} \mathrm{dB}$. SQ: squeezing.

was introduced in channel 1. The local oscillator phase of the homodyne detector was tuned to detect the $x$ quadrature of channel 1. Similarly, in Fig. 4, right column, the error was introduced in channel 9 and the local oscillator phase is locked to the $p$ quadrature. For ease of experimental implementation, only the measurement outcomes of detectors 4 and 8 were fed forward to the error correction step in the case of an error in channel 9. In principle, using the combined outputs of detectors 1 and 4 for $x$ and detectors 7 and 8 for $p$ would yield even higher fidelities.

The quality of the error correction can be assessed through the fidelity $F=\left\langle\psi_{\text {in }}\left|\hat{\rho}_{\text {out }}\right| \psi_{\text {in }}\right\rangle$, where $\left|\psi_{\text {in }}\right\rangle$ represents the input state and $\hat{\rho}_{\text {out }}$ corresponds to the output state of the error correction circuit $^{21-23}$. Here, the fidelity is calculated as

$$
F=\frac{2}{\sqrt{\left(1+4\left\langle\left(\Delta \hat{x}_{\text {out }}\right)^{2}\right\rangle\right)\left(1+4\left\langle\left(\Delta \hat{p}_{\text {out }}\right)^{2}\right\rangle\right)}}
$$

where $\hat{x}_{\text {out }}$ and $\hat{p}_{\text {out }}$ are quadrature operators of the output field. For example, in the case of an error in channel 1, the output quadrature operators become

$$
\begin{aligned}
& \hat{x}_{\text {out }}=\hat{x}_{\text {in }}-\frac{1}{\sqrt{2}} \hat{x}_{\text {an } 1}^{(0)} \mathrm{e}^{-r_{1}} \\
& \hat{p}_{\text {out }}=\hat{p}_{\text {in }}-\frac{1}{\sqrt{6}} \hat{p}_{\text {an } 2}^{(0)} \mathrm{e}^{-r_{2}}
\end{aligned}
$$

where $\hat{x}_{\text {in }}, \hat{p}_{\text {in }}, \hat{x}_{\text {an1 } 1}^{(0)}$ and $\hat{p}_{\text {an2 } 2}^{(0)}$ are quadrature operators of the input field and the ancilla vacuum modes, and $r_{i}$ are squeezing parameters for ancilla $i$. In the ideal case of $r_{i} \rightarrow \infty$, unit fidelity is obtained, with output states approaching the input states. For zero squeezing, equation (3) yields an excess noise of $1 / 2$ and $1 / 6$ for the $x$ and $p$ quadratures, corresponding to 1.76 and $0.67 \mathrm{~dB}$ of output powers, respectively (see Table 2 ).

Equation (2) can be used to translate the measured noise level values from Table 2 into fidelity values. Indeed, for every possible error introduced (in any of the channels), the fidelity after error correction exceeds the maximum values achievable for the scheme in the absence of ancilla squeezing. For example, for an error in mode 1 , a fidelity of $0.88 \pm 0.01$ was achieved (exceeding the 'classical' cutoff of 0.86). Similarly, for an error in channel 9, we obtain a fidelity of $0.86 \pm 0.01$, exceeding a cutoff of 0.82 . (The lower cutoff takes into consideration that only two of the four non-zero components are used.) The improvement over quantumlimited fidelities for errors in any one of the nine channels is the key demonstration of this letter, providing indirect evidence of entanglement-enhanced error correction (see Supplementary Information). By comparison, in the complete absence of any error correction, that is, without reversing displacement errors (including the zero-squeezing case; for an application of such quantumlimited error correction, see Supplementary Information, Part F), fidelity values under $0.007 \pm 0.001$ were obtained. Nonetheless, instead of this absolute improvement, it is the extent to which the classical cutoff is exceeded that quantifies the effectiveness of the non-classical resources.

In the experiment, evidence is obtained for an entanglementenhanced correction of displacement errors; a further increase of the small enhancement of the current implementation would require only higher squeezing levels of the resource states. The scheme should be useful for applications where stochastic errors occur such as free-space communication with fluctuating losses and beam pointing errors ${ }^{17-20}$. The ability to implement QEC in an optical network of this size represents a significant step towards the manipulation and application of large-scale multipartite entanglement for quantum-information processing.

Received 6 February 2008; accepted 29 May 2009; published online 28 June 2009

\section{References}

1. Shor, P. W. Scheme for reducing decoherence in quantum computer memory. Phys. Rev. A 52, R2493-R2496 (1995).

2. Steane, A. M. Error correcting codes in quantum theory. Phys. Rev. Lett. 77, 793-797 (1996).

3. Braunstein, S. L. Quantum error correction for communication with linear optics. Nature 394, 47-49 (1998).

4. Gottesman, D., Kitaev, A. \& Preskill, J. Encoding a qubit in an oscillator. Phys. Rev. A 64, 012310 (2001).

5. Niset, J., Fiurášek, J. \& Cerf, N. J. No-go theorem for Gaussian quantum error correction. Phys. Rev. Lett. 102, 120501 (2009).

6. Cory, D. G. et al. Experimental quantum error correction. Phys. Rev. Lett. 81, 2152-2155 (1998).

7. Leung, D. et al. Experimental realization of a two-bit phase damping quantum code. Phys. Rev. A 60, 1924-1943 (1999).

8. Knill, E. et al. Benchmarking quantum computers: The five-qubit error correcting code. Phys. Rev. Lett. 86, 5811-5814 (2001).

9. Boulant, N. et al. Experimental implementation of a concatenated quantum error-correcting code. Phys. Rev. Lett. 94, 130501 (2005).

10. Chiaverini, J. et al. Realization of quantum error correction. Nature 432, 602-605 (2004)

11. O’Brien, J. L. et al. High-fidelity Z-measurement error encoding of optical qubits. Phys. Rev. A 71, 060303 (2005).

12. Lu, C.-Y. et al. Experimental quantum coding against qubit loss error Proc. Natl Acad. Sci. USA 105, 11050-11054 (2008).

13. Braunstein, S. L. Error correction for continuous quantum variables. Phys. Rev. Lett. 80, 4084-4087 (1998).

14. Lloyd, S. \& Slotine, J.-J. E. Analog quantum error correction. Phys. Rev. Lett. 80, 4088-4091 (1998).

15. Yonezawa, H., Aoki, T. \& Furusawa, A. Demonstration of a quantum teleportation network for continuous variables. Nature 431, 430-433 (2004).

16. van Loock, P. A note on quantum error correction with continuous variables. Preprint at <http://arxiv.org/abs/0811.3616> (2008). 
17. Heersink, J. et al. Distillation of squeezing from non-Gaussian quantum states. Phys. Rev. Lett. 96, 253601 (2006).

18. Dong, R. et al. Experimental entanglement distillation of mesoscopic quantum states. Nature Phys. 4, 919-923 (2008).

19. Hage, B. et al. Preparation of distilled and purified continuous-variable entangled states. Nature Phys. 4, 915-918 (2008).

20. Niset, J., Andersen, U. L. \& Cerf, N. J. Experimentally feasible quantum erasure-correcting code for continuous variables. Phys. Rev. Lett. 101, 130503 (2008)

21. Furusawa, A. et al. Unconditional quantum teleportation. Science 282, 706-709 (1998).

22. Braunstein, S. L. et al. Quantum versus classical domains for teleportation with continuous variables. Phys. Rev. A 64, 022321 (2001).

23. Hammerer, K. et al. Quantum benchmark for storage and transmission of coherent states. Phys. Rev. Lett. 94, 150503 (2005).

\section{Acknowledgements}

This work was partly supported by SCF, GIA, G-COE and PFN commissioned by the MEXT of Japan, and the Research Foundation for Opto-Science and Technology. S.L.B. appreciated discussions with Netta Cohen. P.v.L. acknowledges the DFG for financial support under the Emmy Noether programme. A.F. acknowledges Y. Takeno for preparing the figures. P.v.L. thanks Gerd Leuchs for useful discussions.

\section{Author contributions}

Project planning: T.A., A.F. Experimental work: T.A., G.T., T.K., J.Y. Theoretical work: S.L.B., P.v.L., A.F.

\section{Additional information}

Supplementary information accompanies this paper on www.nature.com/naturephysics. Reprints and permissions information is available online at http://npg.nature.com/ reprintsandpermissions. Correspondence and requests for materials should be addressed to A.F. 